\title{
Directed flow in Au+Au collisions from the RHIC Beam Energy Scan at the STAR experiment
}

\author{
Subhash Singha* \\ (for the STAR Collaboration) \\ Kent State University, Ohio 44242, USA \\ E-mail: subhashercf.rhic.bnl.gov
}

\begin{abstract}
We report results of $v_{1}(y)$ and $d v_{1} / d y$ near mid-rapidity for $\pi^{ \pm}, K^{ \pm}, K_{s}^{0}, p, \bar{p}, \Lambda, \bar{\Lambda}$ and $\phi$ from Beam Energy Scan Au+Au collisions at $\sqrt{s_{N N}}=7.7-200 \mathrm{GeV}$ using the STAR detector at RHIC. The $d v_{1} / d y$ of $\pi^{ \pm}, K^{ \pm}$and $K_{s}^{0}$ mesons remains negative over all beam energies. The $d v_{1} / d y$ of $p$ and $\Lambda$ baryons shows a sign change around $10-15 \mathrm{GeV}$, while net baryons (net p and net $\Lambda$ ) indicate a double sign change. The $d v_{1} / d y$ of $\bar{p}, \bar{\Lambda}$ and $\phi$ show a similar trend for $\sqrt{s_{N N}}>$ 14.5 GeV. For the first time, $v_{1}$ measurements are used to test a quark coalescence hypothesis. Many measurements are found to be consistent with the particles being formed via coalescence of constituent quarks. The observed deviations from that consistency offer a new approach for probing the collision process at the quark level.
\end{abstract}

Critical Point and Onset of Deconfinement - CPOD2017

7-11 August, 2017

The Wang Center, Stony Brook University, Stony Brook, NY

\footnotetext{
* Speaker.
} 


\section{Introduction}

A goal of research at the Relativistic Heavy Ion Collider (RHIC) is to explore deconfined quark-gluon matter [1]. Directed flow $\left(v_{1}\right)$ is one of the observables that is sensitive to the dynamics of the system in early times during the collision process. Both hydrodynamical and transport model calculations indicate that the $v_{1}$ slope at mid-rapidity, especially for baryons, is sensitive to the Equation of State (EoS) of the system [2,3]. Based on hydrodynamics, a minimum in $v_{1}$-slope is proposed as a signature of a first-order phase transition between hadronic matter and quark-gluon plasma (QGP) $[2,3]$. STAR has taken data over a wide range of beam energies $\left(\sqrt{s_{N N}}=7.7-\right.$ $200 \mathrm{GeV}$ ) to explore the QCD phase diagram. One of the goals of this Beam Energy Scan (BES) is to find the softest point of the QCD Equation of State [4]. The $v_{1}$-slope of protons from phaseI of BES [5] changes sign near $\sqrt{s_{N N}} \sim 10 \mathrm{GeV}$ with a minimum around $\sqrt{s_{N N}}=10-20 \mathrm{GeV}$. Also the net-proton $v_{1}$-slope shows a double sign change with a more pronounced minimum in the same energy range. Such a behavior shows a qualitative resemblance to a 3-fluid hydrodynamic model calculation with a 1st-order phase transition [3]. However, current state-of-the-art models are not able to reproduce the basic trend of energy dependence of proton $v_{1}$-slope reported by STAR [6-11]. Moreover, the $v_{1}$ results from models with nominally similar prescriptions for the equation of state differ by an order of magnitude [12]. More theoretical progress is necessary for a definitive interpretation of the data.

Recently we have measured $v_{1}$ for $\Lambda, \bar{\Lambda}, K^{ \pm}, K_{S}^{0}$ and $\phi$ in Beam Energy Scan Au+Au collisions at $\sqrt{s_{N N}}=7.7-200 \mathrm{GeV}$ using the STAR detector [13]. $\Lambda$ hyperon offers the opportunity to study a second baryon species, and can compliment the proton data. Charged kaons and $K_{S}^{0}$ have been observed to depend on the kaon-nucleon potential at the energy of AGS experiments [14]. One can test whether any such effect can be observed in the low energy domain of BES. Furthermore, the $\phi$ meson is interesting because its mass is close to the mass of baryons, but it is a vector meson. So $\phi$ can serve as a probe to study whether the $v_{1}$ depends on flavor (baryon or meson) or mass of a particle species. The $\phi$ is less affected by hadronic interactions than other species. Thus it can be used as a clean probe to study the contribution of the partonic phase to $v_{1}$. The $v_{1}$ measurements with ten different hadron species, having different constituent quarks, will help to disentangle the role of produced and transported quarks in heavy-ion collisions.

\section{Analysis Details}

The analysis was done using $\mathrm{Au}+\mathrm{Au}$ collision data taken by the STAR detector during the years 2010, 2011, and 2014. The STAR detector offers uniform acceptance, full azimuthal coverage and excellent particle identification [15]. The Time Projection Chamber (TPC) [16] is the main detector which performs charged particle tracking near mid-rapidity. The collision centrality is estimated using the charged particle multiplicity in the rapidity region $|\eta|<0.5$, measured with the TPC. We utilize both the Time Projection Chamber (TPC) and Time-of-Flight (ToF) [17] detectors for particles identification. The first-order reaction plane $\left(\Psi_{1}\right)$ is estimated using the two

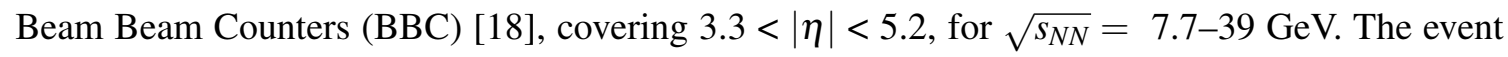
plane resolution from the BBCs deteriorates at $\sqrt{s_{N N}}>39 \mathrm{GeV}$. So we utilize the Zero Degree Calorimeters (ZDC) [19], which cover $|\eta|>6.3$, to estimate $\Psi_{1}$ for $\sqrt{s_{N N}}=62.4$ and $200 \mathrm{GeV}$. 


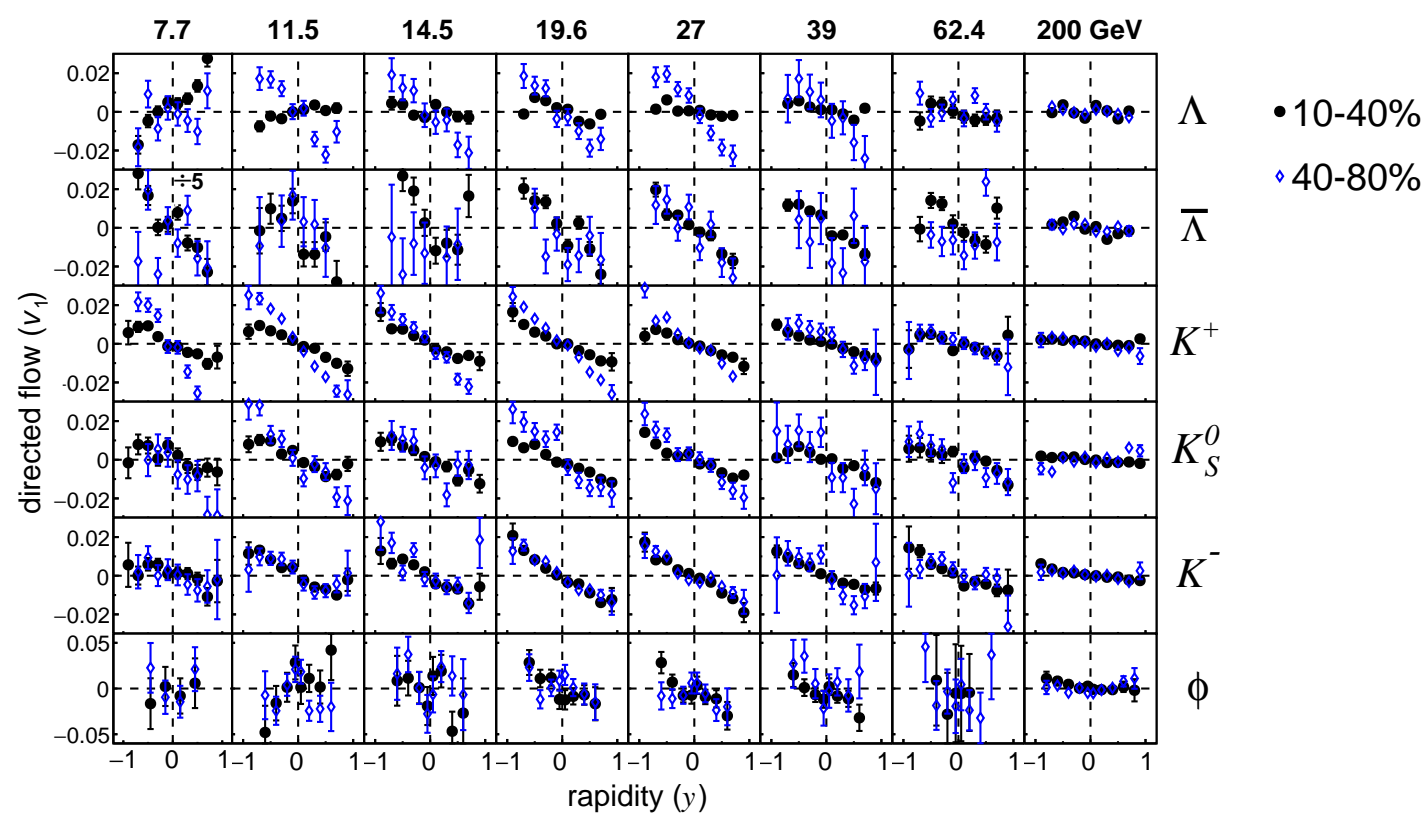

Figure 1: (Color online) Rapidity dependence of directed flow $\left(v_{1}\right)$ for $\Lambda, \bar{\Lambda}, K^{+}, K_{s}^{0}, K^{-}$and $\phi$ in $10-40 \%$ and 40-80\% $\mathrm{Au}+\mathrm{Au}$ collisions at $\sqrt{s_{N N}}=7.7,11.5,14.5,19.6,27,39,62.4$ and $200 \mathrm{GeV}$.

The large $\eta$-gap of these forward detectors (BBC and ZDC) relative to the TPC reduces non-flow contribution in our $v_{1}$ measurements.

\section{Results and discussions}

Figure 1 reports the rapidity dependence of $v_{1}$ for $\Lambda, \bar{\Lambda}, K^{ \pm}, K_{S}^{0}$ and $\phi$ in $10-40 \%$ and $40-80 \%$ central $\mathrm{Au}+\mathrm{Au}$ collisions at $\sqrt{s_{N N}}=7.7,11.5,14.5,19.6,27,39,62.4$ and $200 \mathrm{GeV}$. The data points for $\bar{\Lambda}$ at $7.7 \mathrm{GeV}$ are divided by five in order to present the data in the same vertical scale. It is a common practice to present the strength of $v_{1}(y)$ by its slope at mid-rapidity. In Ref. [5], the $v_{1}$ slope parameter $\left(d v_{1} / d y\right)$ at mid-rapidity was extracted by fitting the data to a cubic function $\left(F_{1} y+F_{3} y^{3}\right)$. In this analysis, poor statistics for $\bar{\Lambda}$ and $\phi$ do not allow a stable cubic fit as done in Ref. [5]. We extract $d v_{1} / d y$ by using a linear function $\left(F_{1} y\right)$ over the rapidity region $|y|<0.8$, except for $\phi$ where the fit is performed for $|y|<0.6$. The energy dependence of the $v_{1}$ slope for $\pi^{ \pm}, K^{ \pm}$and $K_{S}^{0}$ mesons are shown in the top panel in Fig 2. It is observed that all the mesons have negative $v_{1}$ slope. The magnitude of the slope increases with decreasing beam energy. The $K^{+}$slope lies above $K^{-}$at and above $\sqrt{s_{N N}}=11.5 \mathrm{GeV}$ while a reversal of their positions is observed at $\sqrt{s_{N N}}=7.7 \mathrm{GeV}$. The $K_{S}^{0}$ is consistent with the mean of $K^{+}$and $K^{-}$at all studied beam energies. Within the present uncertainties, no distinct mass ordering is observed among the mesons.

The energy dependence of $d v_{1} / d y$ for the baryons and for $\phi$ are presented in the bottom panel of Fig. 2. We observe that $p$ and $\Lambda$ show a sign change near $\sqrt{s_{N N}}=11.5 \mathrm{GeV}$. The $d v_{1} / d y$ of $\bar{p}$ 

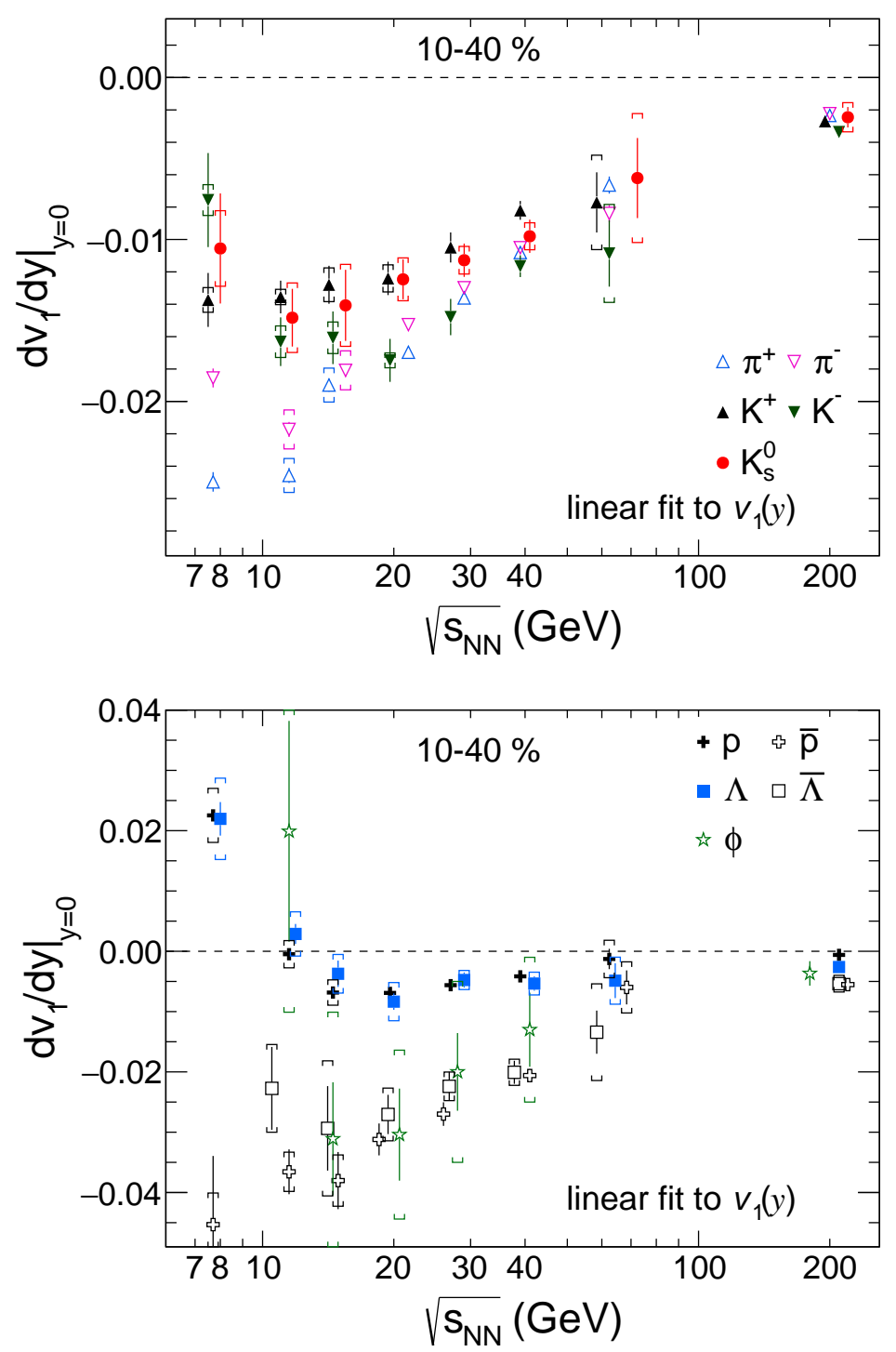

Figure 2: (Color online) Top panel: Beam energy dependence of $d v_{1} / d y$ for $\pi^{ \pm}, K^{ \pm}$and $K_{s}^{0}$ in $10-40 \%$ Au+Au collisions. Bottom panel: Beam energy dependence of $d v_{1} / d y$ for $\Lambda, \bar{\Lambda}, p, \bar{p}$ and $\phi$ in $10-40 \%$ $\mathrm{Au}+\mathrm{Au}$ collisions.

and $\bar{\Lambda}$ remain negative at all beam energies and their magnitude increases with decreasing beam energy. The $\phi$ meson $d v_{1} / d y$ follows the trend of anti-baryons for $\sqrt{s_{N N}}>14.5 \mathrm{GeV}$, while its slope turns towards zero at lower energies. Current statistical and systematic uncertainties for $\phi v_{1}$ at 7.7 and $11.5 \mathrm{GeV}$ are too large to draw any conclusions.

The $v_{1}$ can have contributions from both produced and transported quarks. To disentangle the contribution of the two sources, we define a net particle $v_{1}$ as

$$
F_{\Lambda}=r_{1}(y) F_{\bar{\Lambda}}+\left[1-r_{1}(y)\right] F_{\text {net } \Lambda}
$$




$$
\begin{gathered}
F_{p}=r_{2}(y) F_{\bar{p}}+\left[1-r_{2}(y)\right] F_{\text {net } p}, \\
F_{K^{+}}=r_{3}(y) F_{K^{-}}+\left[1-r_{3}(y)\right] F_{\text {net } K},
\end{gathered}
$$

where $F$ denotes the $d v_{1} / d y$ for each species and $r_{i}(y)$ denotes the rapidity dependent ratios of corresponding anti-particle to particle yields. Figure 3 presents the energy dependence of directed flow slope for net $\Lambda$, net $p$ and net $K$. It is observed that net baryons (net $p$ and net $\Lambda$ ) agree within uncertainties at all energies. The net- $K d v_{1} / d y$ is observed to follow net $p$ and net $\Lambda$ for $\sqrt{s_{N N}}>$ 14.5 GeV, while it diverges from the trend below $14.5 \mathrm{GeV}$.

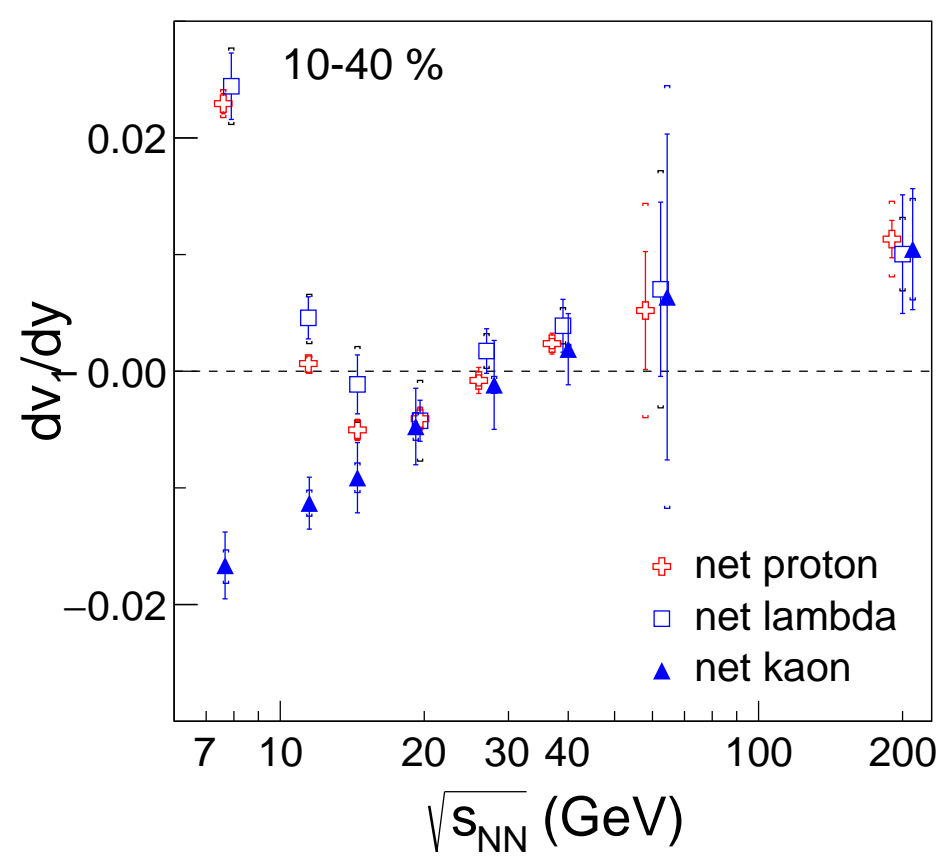

Figure 3: (Color online) Beam energy dependence of net-particle $d v_{1} / d y$ in $10-40 \% \mathrm{Au}+\mathrm{Au}$ collisions.

Number of Constituent Quark (NCQ) scaling has been observed in higher flow harmonics $\left(v_{2}\right.$ and $v_{3}$ ) at RHIC and LHC energies [20-22]. Such scaling is interpreted as evidence of quark degrees of freedom in the early stages of heavy-ion collisions. The $v_{1}$ measurements for ten different particle species allow us to extend tests of a quark coalescence hypothesis using both produced and transported quarks. In a naive quark coalescence picture, we can assume that quarks acquire $v_{1}$ from the medium and then those quarks statistically coalesce to form hadrons. The coalescence

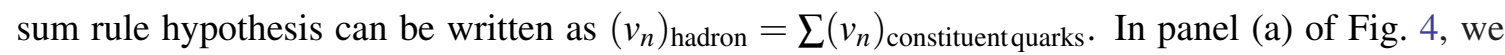
present a test of the quark coalescence sum rule hypothesis utilizing particle species where all the constituent quarks are produced in collisions. The solid black marker presents $d v_{1} / d y$ for $\bar{\Lambda}$. This result is compared with a sum rule hypothesis where we add one third of $\bar{p} d v_{1} / d y$ to $K^{-} d v_{1} / d y$. The factor $\frac{1}{3}$ arises from the assumption that $\bar{u}$ and $\bar{d}$ have the same $v_{1}$. We also assume that the $s$ and $\bar{s}$ have the same $v_{1}$. We observe that this sum rule hypothesis holds for $\sqrt{s_{N N}}=11.5-200 \mathrm{GeV}$, 


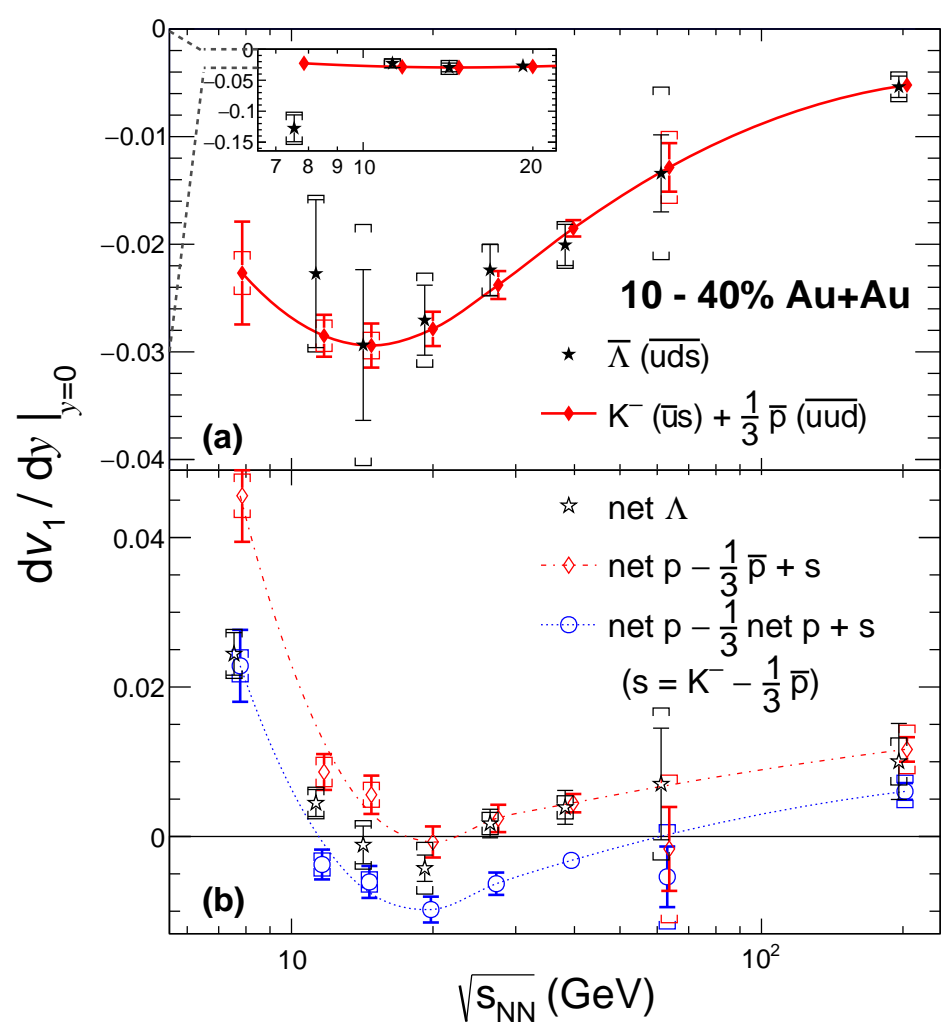

Figure 4: (Color online) Directed flow slope $\left(d v_{1} /\left.d y\right|_{y=0}\right)$ versus $\sqrt{s_{N N}}$ for intermediate-centrality (10$40 \%$ ) Au+Au collisions. Panel (a) compares the observed $\bar{\Lambda}$ slope with the prediction of the coalescence sum rule for produced quarks. The inset shows the same comparison where the vertical scale is zoomed-out; this allows the observed flow for the lowest energy $\left(\sqrt{s_{N N}}=7.7 \mathrm{GeV}\right)$ to be seen. Panel (b) presents two further sum-rule tests, based on comparisons with net- $\Lambda$ measurements. The expression $K^{-}(\bar{u} s)-\frac{1}{3} \bar{p}(\overline{u u d})$ represents the $s$ quark flow; there is no corresponding clear-cut expression for transported $u$ and $d$ quarks.

while it deviates at $7.7 \mathrm{GeV}$. The deviation at the lowest energy indicates that the above-mentioned assumptions do not hold at $\sqrt{s_{N N}}=7.7 \mathrm{GeV}$.

Panel (b) in Fig. 4 presents tests of the coalescence sum rule hypothesis using $u$ and $d$ quarks, which could be transported or produced. We utilize net-particle $v_{1}$ to test the coalescence hypothesis in this less straightforward situation. The fraction of transported quarks among the constituent quarks of net particles is larger than in particles roughly in proportion to $N_{\text {particle }} / N_{\text {netparticle }}$. Here we assume that all the transported quarks are contained in net particles. In panel (b) of Fig. 4, open black symbols present net- $\Lambda d v_{1} / d y$. The contribution of transported quarks will increase in net $\Lambda$ at low beam energies, where the $u$ and $d$ quarks are more likely to be transported from the colliding nuclei. In the high energy limit, the constituent quarks in net $\Lambda$ are more likely to be produced in the collision. The net- $\Lambda$ result is compared with two different coalescence sum rule calculations. In the first calculation, shown by open red markers, we replace one of the $u$ quarks in net proton with a $\bar{u}$ quark, and also add the $d v_{1} / d y$ contribution from an $s$ quark. The $s$ quark contribution is obtained by subtracting one third of $\bar{p} d v_{1} / d y$ from $K^{-} d v_{1} / d y$. The first sum rule calculation is 
consistent with net $\Lambda$ within errors for $\sqrt{s_{N N}}>19.6 \mathrm{GeV}$. As the contribution of transported quarks increases in net $\Lambda$ at lower energies, the first sum rule calculation deviates as we scan further below 19.6 GeV. In the second sum rule test (shown by blue open markers), we add one $s$ quark $d v_{1} / d y$ to two-thirds of net- $p d v_{1} / d y$. Here we assume that the constituent quarks in net $p$ are dominated by transported quarks. We find that this second sum rule calculation agrees with net $\Lambda$ at the lowest beam energy and it deviates with increasing beam energy.

\section{Conclusions and outlook}

In summary, we reported the measurement of directed flow $\left(v_{1}\right)$ near midrapidity for $\pi^{ \pm}, K^{ \pm}$, $K_{s}^{0}, p, \bar{p}, \Lambda, \bar{\Lambda}$ and $\phi$ spanning eight beam energies over the range $\sqrt{s_{N N}}=7.7$ to $200 \mathrm{GeV}$. We observe that the proton and $\Lambda d v_{1} / d y$ agree within errors and they change sign near $\sqrt{s_{N N}}=11.5 \mathrm{GeV}$. The slopes for $\pi^{ \pm}, K^{ \pm}, K_{s}^{0}, \bar{\Lambda}$ and $\bar{p}$ are negative at all available beam energies. The $K_{s}^{0}$ is consistent with the mean of $K^{+}$and $K^{-}$at all beam energies. The $\phi d v_{1} / d y$ is negative and follows the same trend as $\bar{p}$ and $\bar{\Lambda}$ for $\sqrt{s_{N N}}>14.5 \mathrm{GeV}$. Net-particle $d v_{1} / d y$ for $p, \Lambda$ and $K$ agree with each other at and above $\sqrt{s_{N N}}=14.5 \mathrm{GeV}$, but net $K$ diverges at 11.5 and $7.7 \mathrm{GeV}$. A quark coalescence sum rule hypothesis is tested using $v_{1}$ measurements. Produced quarks follow coalescence sum-rule behavior at $11.5-200 \mathrm{GeV}$, but strongly deviate at $7.7 \mathrm{GeV}$. This offers a new approach to probe the heavy-ion collision process at the quark level.

The STAR collaboration will upgrade detectors in phase-II of the RHIC Beam Energy Scan [23]. The upgrade of the inner TPC will not only improve particle identification but will also enhance forward rapidity coverage. A new endcap Time-of-Flight detector (eTOF) will further extend particle identification. Furthermore, future measurements will greatly benefit from a new Event Plane Detector (EPD), which will improve the first-order event plane resolution by a factor of two. The EPD will also provide an independent collision centrality determination. These detector upgrades and increased statistics in BES-II will significantly improve the precision and quality of many measurements.

Recently, a model calculation [24] predicted that the early transient magnetic field can induce a larger $v_{1}$ for heavy quarks than for light quarks. The model calculation also suggests an opposite sign of $d v_{1} / d y$ for charm $(c)$ and anti-charm $(\bar{c})$ quarks. This offers hope that heavy quarks will offer a new approach to study the early electromagnetic field. STAR has collected over 2 billion events with the Heavy Flavor Tracker (HFT) detector during the years 2014 and 2016. The HFT has demonstrated excellent performance in reconstructing heavy flavor hadrons. We also look forward to the measurement of $D^{0}(\bar{u} c)$ and $\overline{D^{0}}(u \bar{c})$ directed flow utilizing both the HFT and ZDC detectors in STAR to probe the early transient magnetic field.

\section{Acknowledgments}

This work is supported in part by the US Dept. of Energy under grant DE-FG02-89ER40531.

\section{References}

[1] J. Adams et al. (STAR Collaboration), Nucl. Phys. A 757, 102 (2005). 
[2] D. H. Rischke et al., Heavy Ion Phys. 1, 309 (1995).

[3] H. Stöcker, Nucl. Phys. A 750, 121 (2005).

[4] M. M. Aggarwal et al. (STAR collaboration), arXiv:1007.2613; STAR Note SN0493 (2009).

[5] L. Adamczyk et al. (STAR collaboration), Phys. Rev. Lett. 112, 162301 (2014).

[6] J. Steinheimer, J. Auvinen, H. Petersen, M. Bleicher and H. Stöcker, Phys. Rev. C 89, 054913 (2014).

[7] V. P. Konchakovski, W. Cassing, Y. B. Ivanov and V. D. Toneev, Phys. Rev. C 90, 014903 (2014).

[8] W. Cassing, V. P. Konchakovski, A. Palmese, V. D. Toneev and E. L. Bratkovskaya, Proc. 3rd Int. Conf. on New Frontiers in Physics, Kolymbari, Crete, 2014, EPJ Web Conf. 95, 01004 (2015).

[9] Yu. B. Ivanov and A. A. Soldatov, Phys. Rev. C 91, 024915 (2015).

[10] Y. Nara, H. Niemi, A. Ohnishi and H. Stöcker, Phys. Rev. C 94, 034906 (2016).

[11] Y. Nara, H. Niemi, J. Steinheimer and H. Stöcker, Phys. Lett. B 769, 543 (2017).

[12] S. Singha, P. Shanmuganathan and D. Keane, Adv. High Energy Phys. 2016, 2836989 (2016).

[13] L. Adamczyk et al. (STAR Collaboration), arXiv:1708.07132 (Accepted for publication in Phys. Rev. Lett.)

[14] G. Q. Li, C. M. Ko and B. A. Li, Phys. Rev. Lett. 75, 235 (1995); P. Chung et al. (E895 Collaboration), Phys. Rev. Lett. 85, 940 (2000).

[15] K. H. Ackermann et al., Nucl. Instr. Meth. A 499, 624 (2003).

[16] M. Anderson et al., Nucl. Instr. Meth. A 499, 659 (2003).

[17] W. J. Llope (for the STAR TOF Group) Nucl. Instr. Meth. in Phys. Research B 241, 306 (2005).

[18] C. A. Whitten (STAR Collaboration), AIP Conf. Proc. 980, 390 (2008).

[19] G. Wang, PhD thesis, Kent State University, 2006; https://drupal.star.bnl.gov/STAR/theses.

[20] J. Adams et al. ( STAR Collaboration), Phys. Rev. Lett. 92, 052302 (2004); B. Abelev et al. (STAR Collaboration), Phys. Rev. C 75, 054906 (2007); J. Adams et al. ( STAR Collaboration), Phys. Rev. C 72, 014904 (2005); B. I. Abelev et al. (STAR Collaboration), Phys. Rev. Lett. 99, 112301 (2007).

[21] S. S. Adler et al. ( PHENIX Collaboration), Phys. Rev. Lett. 91, 182301 (2003); S. Afanasiev et al. (PHENIX Collaboration), Phys. Rev. Lett. 99, 052301 (2007); A. Adare et al. ( PHENIX Collaboration), Phys. Rev. Lett. 98, 162301 (2007); A. Adare et al. ( PHENIX Collaboration), Phys. Rev. C 85, 064914 (2012).

[22] B. Abelev et al. (ALICE Collaboration), JHEP 06, 190 (2015); K. Aamodt et al. (ALICE Collaboration), Phys. Rev. Lett. 105, 252302 (2010).

[23] STAR collaboration, STAR Note SN0598 (2014).

[24] S. Das, S. Plumari, S. Chatterjee, J. Alam, F. Scardina and V. Greco, Phys. Lett. B 768, 260-264 (2017). 